\title{
Ocorrência de distúrbio musculoesquelético em pescadoras artesanais/ marisqueiras na Baía de Todos os Santos: uma análise sobre horas dedicadas ao trabalho
}

\author{
Occurrence of musculoskeletal disorders in artisanal /shellfish fisherwomen in Baía de \\ Todos os Santos: an analysis of hours dedicated to work
}

\author{
Juliana dos Santos Müller', Rita de Cássia Franco Rêgo², Carlos Mauricio Cardeal Mendes ${ }^{3 *}$ \\ ${ }^{1}$ Doutoranda do Programa de Pós-graduação em Processos Interativos de Órgãos e Sistemas, Universidade \\ Federal da Bahia; Professora do Instituto Federal de Santa Catarina/ Departamento de Saúde e Serviços - Campus \\ Florianópolis ${ }^{2}$ Doutora em Saúde Coletiva. Professora do Programa de Pós-graduação em Saúde, Ambiente \\ e Trabalho, Faculdade de Medicina, Universidade Federal da Bahia ${ }^{3}$ Doutor em Saúde Coletiva. Professor do \\ Programa Pós-graduação em Processos Interativos de Órgãos e Sistemas, Universidade Federal da Bahia
}

\begin{abstract}
Resumo
Introdução: os distúrbios musculoesqueléticos são caracterizados por comprometimentos inflamatórios e/ou degenerativos, em diversos segmentos corporais, e atingem diversos grupos de trabalhadores, no entanto, ainda existem poucas evidências científicas que verificam possíveis relações desses distúrbios e horas de trabalho diárias dos pescadores artesanais. Objetivo: analisar as horas diárias dedicadas ao trabalho de pescadora e a prevalência de distúrbio musculoesquelético em regiões de membros superiores (ombro) e pescoço. Metodologia: realizou-se um estudo de corte transversal, envolvendo amostra probabilística da população de pescadoras artesanais/marisqueiras do município de Saubara, totalizando 209 pessoas. Utilizaram-se instrumentos validados para população brasileira, tais como: o Nordic Musculoskeletal Questionnaire, Job Content Questionnaire e Demandas físicas adaptadas para o trabalho. Resultados: evidenciou-se a prevalência de distúrbios musculoesqueléticos em região de pescoço ou ombro, em $71,2 \%$ ( $n=146$ ) das marisqueiras. Ressalta-se que não houve interação entre as variáveis horas trabalhadas/dia e idade, com o p-valor $=0,1807$. Verificaram-se associações positivas entre as marisqueiras que trabalhavam mais que $11 \mathrm{~h}$ por dia com razão de prevalência de 1,23 e intervalo de confiança $95 \%:[1,04 ; 1,45]$ em relação às que trabalhavam menos que $11 \mathrm{~h}$, bem como a razão de prevalência de 1,38 e intervalo de confiança $95 \%:[1,15 ; 1,65]$ nas pescadoras com idade superior a 38 em comparação com as mais jovens. Desta forma denotando a degradação da saúde com a carga exaustiva de trabalho.

Palavras chaves: Distúrbios musculoesqueléticos. Pescadores artesanais. Razão de prevalência.
\end{abstract}

\begin{abstract}
Introduction: musculoskeletal disorders are characterized by inflammatory and/or degenerative impairments in several body segments and reach different groups of workers; however, there is still little scientific evidence to verify the possible relationships between MSD and daily working hours of artisanal fishermen. Objective: to analyze the daily hours dedicated to work as a fisherwoman and prevalence of musculoskeletal disorders in regions of upper limbs (shoulder) and neck. Methodology: a cross-sectional study was carried out involving a probabilistic sample of the population of artisanal/shellfish fisherwomen in the municipality of Saubara, for a total of 209 individuals. Validated instruments were used for the Brazilian population, such as: the Nordic Musculoskeletal Questionnaire, the Job Content Questionnaire and the Physical Demands adapted to this work. Results: the prevalence of musculoskeletal disorders in the neck or shoulder region was found in $71.2 \%(n=146)$ of shell fisherwomen. It should come to attention that there was no interaction between the variables worked hours/day and age, with $p$-value $=0.1807$. There were positive associations among the shellfish workers that worked more than $11 \mathrm{~h}$ with the prevalence ratio of 1.23 and $95 \%$ confidence interval: [1.04; 1.45$]$ than those who worked less than 11 hours, as well as the prevalence ratio of 1.38 and $95 \%$ confidence interval: $[1.15 ; 1.65]$ in fisherwoman aged over 38 compared to younger ones. Thus denoting health degradation with an exhaustive workload.

Keywords: Musculoskeletal Disorders. Artisanal Fisherwomen. Prevalence Ratio
\end{abstract}

\section{INTRODUÇÃO}

Nas últimas décadas, muitos estudos científicos demonstraram a alta prevalência de distúrbios musculoesqueléticos (DME) em trabalhadores de países indus-

Correspondente/Corresponding: *Carlos Mauricio Cardeal Mendes End: : Av. Reitor Miguel Calmon S/N sala 413 Canela Salvador-Ba CEP: 40231-300 - E-mail: mcardeal@ufba.br — Tel: (71)99974-9504 trializados e em desenvolvimento (FERNANDES et al., 2016). No Brasil, a presença de sintomas relacionados a tais distúrbios ocupa o segundo lugar nos afastamentos e despesas previdenciárias relacionadas ao trabalho, o que poderá repercutir em incapacidade temporária ou permanente desses trabalhadores (ALMEIDA; BARBOSA-BRANCO, 2011). 
Nesta perspectiva, destaca-se uma das atividades laborais mais antigas feitas pelo homem, a pesca artesanal, um trabalho secular, perigoso e desgastante, no qual as medidas de proteção social a esse segmento de trabalhadores têm importância primordial, pois no Brasil são produzidos cerca de 1.240 .000 pescados por ano e a pesca artesanal é responsável por $45 \%$ da produção nacional; no entanto, não existem dados oficiais brasileiros desde o fim do Ministério da Pesca e Aquicultura e criação da Secretaria da Pesca (BRASIL. MINISTÉRIO DA PESCA E AQUICULTURA, 2012). Segundo a Food and Agricultural Organization (2016), o Brasil ocupa o quarto lugar na produção de pescado na região da América Latina e Caribe.

A presença do DME se destaca dentre os agravos inerentes a essa atividade, caracterizados por comprometimentos inflamatórios e/ou degenerativos de tendões, fáscias, músculos, articulações, bursas, ligamentos, circulação, vasos sanguíneos, nervos periféricos e raízes nervosas, em diferentes segmentos corporais como a região do pescoço e ombro, cotovelos, antebraços, punhos, mãos, região lombar e segmentos dos membros inferiores (BRASIL. MINISTÉRIO DA SAÚDE, 2001; PUNNETT; WEGMAN, 2004).

Tais quadros clínicos se distinguem pela ocorrência de diversos sintomas, concomitantes ou não, de aparecimento insidioso (BRASIL. MINISTÉRIO DA SAÚDE, 2001, 2006). A presença de DME nos trabalhadores da pesca é condição impactante sobre a saúde e torna-se muito complexa devido à condição informal dessa atividade, corroborando a ausência de identificação, de medidas de promoção, de prevenção, de assistência à saúde e de reabilitação desse grande contingente de trabalhadores.

Nesse contexto, ressalta-se que existem poucos estudos que verificam possíveis relações de DME e trabalho com grupos de pescadoras artesanais/marisqueiras no Brasil. As marisqueiras brasileiras, em especial na região Nordeste, realizam todas as etapas do seu trabalho de forma artesanal e tradicional. A coleta de crustáceos e moluscos, ao longo da costa brasileira, pode ocasionar agravos à saúde dessas trabalhadoras (RIOS; REGO; PENA, 2011), as quais retiram do mar, mangue, rio ou praia a sua subsistência, estando sujeitas à sobrecarga muscular em diversas regiões do corpo (PENA; FREITAS; CARDIM, 2011). Já foi relatado que as marisqueiras de Saubara apresentam menor qualidade de vida relacionada à saúde, em comparação com a população brasileira e com marisqueiras da Galícia (MÜLLER et al., 2016).

Em pesquisa realizada em Saubara, município da Baía de Todos os Santos (BTS), cuja população vive quase exclusivamente da pesca artesanal, identificaram-se, dentre as principais demandas da comunidade de pescadoras artesanais/marisqueiras, queixas relacionadas ao DME, atribuído às condições do trabalho (FALCÃO et al., 2015).

Ressalta-se que DME de pescoço e membros superiores têm causa multifatorial (ARMSTRONG et al., 1993; BONGERS et al., 2006; LIN et al., 2001; NATIONAL RESEARCH COUNCIL; INSTITUTE OF MEDICINE, 2001, p.
49; WORLD HEALTH ORGANIZATION, 1985, p. 9) e uma natureza complexa de interação entre exposição, dose, capacidade e resposta (ARMSTRONG et al., 1993). Cada distúrbio clínico representa uma interação complexa entre a pessoa afetada e uma variedade de determinantes que influenciam na resposta individual (NATIONAL RESEARCH COUNCIL; INSTITUTE OF MEDICINE, 2001, p. 49).

Os fatores de riscos descritos na literatura para o desenvolvimento dos DME incluem: idade, gênero, Índice de Massa Corpórea (IMC), hábitos pessoais como o fumo, além das atividades realizadas fora do local de trabalho (trabalho doméstico e prática de atividade física), todos considerados fontes de confundimento (NATIONAL RESEARCH COUNCIL; INSTITUTE OF MEDICINE, 2001, p. $18,25)$. Algumas comorbidades podem contribuir para a sintomatologia do DME, como por exemplo, a artrite reumatoide e o diabete melito (LIN et al., 2001).

Entre os fatores de exposição para presença de DME, relacionados e não relacionados ao trabalho, destacam-se: os aspectos físicos, organizacionais e sociais, relacionados à ocupação e ao ambiente de trabalho, ademais das características físicas e psicológicas individuais (NATIONAL RESEARCH COUNCIL; INSTITUTE OF MEDICINE, p. 16, 2001).

Dessa forma, estudos epidemiológicos com essas trabalhadoras são necessários para verificar a presença de associação entre os fatores de risco e a presença de distúrbio musculoesquelético (DME), em especial na unidade funcional que compõe os membros superiores (ombro) e região de pescoço. Para tanto, este artigo tem como objetivo averiguar o histórico laborativo, caracterizado pelas horas diárias dedicadas ao trabalho de pescadora/ marisqueira (considerando todas as etapas do trabalho), e a ocorrência de DME em membros superiores e pescoço.

\section{METODOLOGIA}

Trata-se de um estudo de corte transversal, realizado com 209 pescadoras artesanais/marisqueiras. Como instrumentos para levantamento de dados, utilizaram-se a versão brasileira do Questionário de conteúdo do trabalho (Job Content Questionnaire - JCQ) (ARAÚJO; KARASEK, 2008; KARASEK, 1985; KARASEK et al., 1998) e do Questionário nórdico (Nordic Musculoskeletal Questionnaire - NMQ) (KUORINKA et al., 1987; KUORINKA; FORCIER, 1995) e um questionário contendo as demandas físicas adaptadas para o trabalho (FERNANDES, 2004).

\section{Amostragem e critério de inclusão}

A amostragem foi feita de forma aleatória, simples e sem reposição, sendo realizado um sorteio dos indivíduos, tomando por base o total das marisqueiras inscritas na Associação de Pescadoras Artesanais/Marisqueiras de Saubara. A amostra mínima calculada foi de 203 marisqueiras, sendo ao final constituída de 209 marisqueiras, 3\% a mais da amostra mínima. Dentre os critérios de inclusão para participar do estudo, estabeleceram-se: 
ser do sexo feminino, pois esta atividade é exercida nessa comunidade principalmente por mulheres; ter idade maior ou igual a 18 anos; e estar exercendo a atividade há pelo menos um ano. As trabalhadoras sorteadas que não estavam exercendo a atividade de mariscagem tiveram a oportunidade de participar, caso justificassem o afastamento por doença com possível relação ao DME, a fim de minimizar o efeito do viés de sobrevivência do trabalhador sadio.

\section{Variável resposta}

Distúrbio músculo esquelético em pescoço e ombro (DME em P/O) é um desfecho dicotômico, analisado de acordo com sua presença (sim) ou ausência (não). O DME foi definido como o registro de dor ou desconforto de acordo com a região, nos últimos 12 meses, com duração mínima de uma semana ou frequência mínima mensal, não causada por lesão aguda. Os sintomas deveriam estar relacionados, pelo menos, a um dos seguintes itens: grau de severidade $\geq 3$ em uma escala de 0 a 5 (nenhum desconforto a dor insuportável); busca de atenção médica pelo problema; ausência ao trabalho (oficial ou não); ou mudança de trabalho por restrição de saúde (FERNANDES; CARVALHO; ASSUNÇÃO, 2011; KUORINKA et al.,1987; KUORINKA; FORCIER, 1995). Os DME foram analisados de acordo com a unidade funcional pescoço/ombro. A análise do DME de acordo com essa classificação é considerada por alguns autores (ANDERSEN; HAAHR; FROST, 2007; WANG et al., 2007).

\section{Exposição principal}

A exposição principal foi definida como as horas diárias dedicadas ao trabalho de pescadora/marisqueira e foram avaliadas de acordo com a quantidade autorreferida de horas trabalhadas, apenas com as funções relacionadas com o trabalho da marisqueira. Foram consideradas como atividades relacionadas à preparação do material (realizada em casa antes de ir para a coleta), caminhada até o local de trabalho, coleta de marisco (realizada no mar, praia, mangue e/ou rio), transporte do marisco coletado de volta para casa, lavagem do marisco, coleta de lenha, cozimento do marisco, cata do marisco (separação da casca da polpa) e embalagem/armazenamento. As horas diárias de trabalho foram dicotomizadas de acordo com o terceiro quartil em 0 ( $\leq 11$ horas/dia) e 1 ( $>11$ horas/ dia). Esta exposição principal foi definida pela ausência de estudos com trabalhadores informais, refletindo esse desfecho de interesse, isto é, horas de trabalho por dia.

\section{Covariáveis}

Incluíram-se as variáveis descritas na literatura como potenciais confundidores para a associação de interesse e os principais fatores de risco para DME. Entre elas estão: idade, escolaridade, sobrepeso/obesidade, sexo feminino, prática de atividade física no tempo livre, diagnóstico médico de diabetes, diagnóstico médico de artrite reumatoide, demandas físicas e psicossociais (LIN et al., 2001; NATIONAL RESEARCH COUNCIL; INSTITUTE OF MEDICINE, 2001).

Embora estudos relevantes sobre os principais fatores de risco para DME de membros superiores não demonstrem que o tempo de trabalhos em anos (ao longo da vida) é uma variável potencialmente confundidora, os autores a incluíram no plano de análise por ser considerada importante para explicar o desfecho de interesse. A ocorrência de DME é considerada uma interação complexa dos hábitos de vida e caraterísticas pessoais e, neste contexto, seguiu-se o raciocínio de inclusão da variável horas de trabalho semanais dedicadas ao trabalho doméstico (dicotomizada pelo quartil 1). Todas as informações foram obtidas a partir do autorrelato, com exceção do peso e altura, que foram aferidos por entrevistadores treinados.

Para a variável escolaridade foi feita a dicotomização em nível de escolaridade menor que segundo grau incompleto (1) e maior ou igual ao segundo grau incompleto (0). O Índice de Massa Corpórea (IMC) foi avaliado de acordo com a classificação internacional de baixo peso, sobrepeso e obesidade para a população adulta (WORLD HEALTH ORGANIZATION, 2004). Para as análises estatísticas, os valores correspondentes para baixo peso e peso normal foram unidos, subdividindo o IMC em duas categorias: baixo peso e peso normal (IMC $\left.\leq 24,99 \mathrm{Kg} / \mathrm{m}^{2}\right)$ e sobrepeso/ obesidade (IMC $\left.\geq 25,00 \mathrm{Kg} / \mathrm{m}^{2}\right)$. A atividade física no tempo livre foi definida como correr, fazer ginástica, nadar, jogar futebol, andar de bicicleta, caminhar, cuidar da horta ou do quintal, pelo menos três vezes na semana, por no mínimo 30 minutos.

As demandas físicas consideradas foram pressão física e força muscular ( $0:<2$ e $1: \geq 2$, numa escala de 0 a 5 com âncoras nas extremidades). As demandas psicossociais foram dicotomizadas pelas medianas em alta exposição (demanda maior que 34, controle igual ou menor que 66 e suporte social igual ou menor que 13) e baixa exposição (demanda igual ou menor que 34 , controle maior que 66 e suporte social maior que 13). Pelo menos dois desses critérios tiveram que ser atendidos para a marisqueira ser classificada em cada grupo (DEVEREUX; VLACHONIKOLIS; BUCKLE, 2002).

\section{Plano de análise}

Calcularam-se as medidas de dispersão e tendência central para as variáveis contínuas e as medidas de frequência para as categóricas. Após a análise descritiva dos dados, as variáveis idade e horas de trabalho foram dicotomizadas. As variáveis idade, tempo de trabalho em anos, horas diárias dedicadas à mariscagem e horas semanais dedicadas à atividade doméstica foram dicotomizadas pelos quartis que melhor se relacionavam com as variáveis-resposta.

A pré-seleção das variáveis independentes para entrada no modelo de regressão linear generalizado inicial 
foi baseada na plausibilidade e nas análises bivariadas, considerando um valor de $p$ inferior a 0,20 para entrada no modelo, no teste qui-quadrado. Os modelos finais foram obtidos pelo método de seleção backward, com base no teste da razão de verossimilhanças, considerando um nível de significância de $5 \%$.

Para a estimativa direta das razões de prevalência ajustadas, optou-se pela realização de modelagem por regressão múltipla de Poisson (ZOU, 2004), associada à estimativa da matriz de covariância, consistente com a heteroscedasticidade das estimativas dos coeficientes do modelo (MACKINNON; WHITE, 1985), para produzir uma estimativa robusta dos erros-padrão, no intuito de prevenir alguma violação do pressuposto de igualdade entre média e variância da distribuição de Poisson e consequente estimativa mais adequada dos valores de $p$ do modelo. Para o cálculo dos intervalos de confiança a 95\%, acresceu-se o emprego do método Delta (MANDEL, 2013). A bondade de ajuste do modelo foi avaliada pela análise da relação entre os desvios nulos (null deviance) e pelos desvios residuais (residual deviance), enquanto a qualidade do modelo foi avaliada pelo critério de informação de Akaike (AIC) (SAKAMOTO; ISHIGURO; KITAGAWA, 1986).
Os dados foram analisados mediante o pacote estatístico R 3.4.3 (R CORE TEAM, 2018).

\section{RESULTADOS}

Participaram deste estudo 209 pescadoras artesanais/ marisqueiras, com predomínio de 50,73\% (n=104) de muIheres acima de 38 anos. A média de idade foi de 39,6 anos (DP $=11,5$ anos) e a média de horas trabalhadas foi de 8,7 horas $(D P=3,1)$ e, em sua grande maioria $(74,15 \%)$, com escolaridade menor que o $2^{\circ}$ grau incompleto. Conforme caso estabelecido para reconhecimento dos sintomas de DME, este artigo evidenciou a prevalência de distúrbios musculoesqueléticos em $71,2 \%(n=146)$ das marisqueiras, em região de pescoço ou ombro (DME P/O) .

Pôde-se observar ainda que, entre as marisqueiras que trabalham mais de 11 horas por dia, 86,11\% tem DME P/O (Tabela 1). Em todas as variáveis, mesmo nos grupos considerados não expostos, verificam-se elevadas prevalências de DME P/O em mulheres negras e pardas $(96,2 \%)$, escolaridade máxima no ensino fundamental (77\%) e estado civil predominante é o de casadas (64,6\%).

Tabela 1 - Distribuição de frequência simples das variáveis estudadas, prevalência e razão de prevalência (RP) de DME no pescoço ou ombro $\left(n=205^{a}\right)$, segundo exposição principal e potenciais confundidores em uma amostra de Marisqueiras de Saubara, BA, 2013.

\begin{tabular}{|c|c|c|c|}
\hline Característica & $n(\%)$ & DME P/O & $\begin{array}{c}\text { RP [IC]95\% (modelo } \\
\text { não ajustado) }\end{array}$ \\
\hline Prevalência de distúrbio musculoesquelético em pescoço e ombro & $71,2 \%(n=146)$ & - & - \\
\hline \multicolumn{4}{|l|}{ Horas de trabalho/dia (exposição principal) } \\
\hline$\geq 11 \mathrm{~h} / \mathrm{dia}$ & $36(17,56)$ & $31(86,11)$ & $1,26[1,07-1,50]$ \\
\hline$<11 \mathrm{~h} / \mathrm{dia}$ & $169(82,44)$ & $115(68,05)$ & 1 \\
\hline p-valor & & $0,03^{*}$ & \\
\hline \multicolumn{4}{|l|}{ Idade } \\
\hline$\geq 38$ anos & $104(50,73)$ & $86(82,69)$ & $1,39[1,16-1,67]$ \\
\hline$<38$ anos & $101(49,27)$ & $60(59,41)$ & 1 \\
\hline p-valor & & $0,00^{*}$ & \\
\hline \multicolumn{4}{|l|}{ Escolaridade } \\
\hline$<2^{\circ}$ incompleto & $152(74,15)$ & $113(74,34)$ & $1,19[0,95-1,50]$ \\
\hline$\geq 2^{\circ}$ incompleto & $53(25,85)$ & $33(62,26)$ & 1 \\
\hline p-valor & & 0,09 & \\
\hline \multicolumn{4}{|l|}{ Tempo de trabalho em anos } \\
\hline$>26$ anos & $98(47,80)$ & $77(78,57)$ & $1,22[1,02-1,45]$ \\
\hline$\leq 26$ anos & $107(52,20)$ & $69(64,49)$ & 1 \\
\hline p-valor & & $0,03^{*}$ & \\
\hline \multicolumn{4}{|l|}{ Horas de trabalho doméstico/semana } \\
\hline$>7 h$ & $157(76,59)$ & $114(72,61)$ & $1,09[0,87-1,36]$ \\
\hline$\leq 7 h$ & $48(23,41)$ & $32(66,67)$ & 1 \\
\hline p-valor & & 0,43 & \\
\hline \multicolumn{4}{|l|}{ IMC } \\
\hline$\geq 25,0 \mathrm{Kg} / \mathrm{m}^{2}$ & $141(68,78)$ & $100(70,92)$ & $0,99[0,82-1,19]$ \\
\hline
\end{tabular}




\begin{tabular}{|c|c|c|c|}
\hline Característica & $n(\%)$ & DME P/O & $\begin{array}{l}\text { RP [IC]95\% (modelo } \\
\text { não ajustado) }\end{array}$ \\
\hline$\leq 24,9 \mathrm{Kg} / \mathrm{m}^{2}$ & $64(31,22)$ & $46(71,88)$ & 1 \\
\hline p-valor & & 0,89 & \\
\hline \multicolumn{4}{|l|}{ Atividade física } \\
\hline Não (< 3x semana/ 30min) & $133(64,88)$ & $97(72,93)$ & $1,07[0,89-1,29]$ \\
\hline $\operatorname{Sim}(\geq 3 x$ semana/30min) & $72(35,12)$ & $49(68,06)$ & 1 \\
\hline p-valor & & 0,46 & \\
\hline \multicolumn{4}{|l|}{ Força $^{b}$} \\
\hline$\geq 2$ & $191(93,17)$ & $135(70,68)$ & $0,90[0,67-1,20]$ \\
\hline$<2$ & $14(6,83)$ & $11(78,57)$ & 1 \\
\hline p-valor & & 0,53 & \\
\hline \multicolumn{4}{|l|}{ Pressão física ${ }^{b}$} \\
\hline$\geq 2$ & $143(69,76)$ & $102(71,33)$ & $1,00[0,83-1,22]$ \\
\hline$<2$ & $62(30,24)$ & $44(70,97)$ & 1 \\
\hline p-valor & & 0,96 & \\
\hline \multicolumn{4}{|l|}{ Demanda psicossocial } \\
\hline Alta & $103(50,24)$ & $68(66,02)$ & $0,86[0,72-1,03]$ \\
\hline Baixa & $102(49,76)$ & $78(76,47)$ & 1 \\
\hline $\mathrm{p}$-valor & & 0,10 & \\
\hline \multicolumn{4}{|l|}{ Artrite } \\
\hline Sim & $22(10,73)$ & $20(90,91)$ & $1,32[1,12-1,56]$ \\
\hline Não & $183(89,27)$ & $126(68,85)$ & 1 \\
\hline p-valor & & $0,03^{*}$ & \\
\hline \multicolumn{4}{|l|}{ Diabetes } \\
\hline Sim & $14(6,83)$ & $12(85,71)$ & $1,22[0,97-1,54]$ \\
\hline Não & $191(93,17)$ & $134(70,16)$ & 1 \\
\hline $\mathrm{p}$-valor & & 0,22 & \\
\hline
\end{tabular}

Fonte: Dados da pesquisa.

Legenda: ${ }^{a} O$ " $n$ " foi diferente, pois foi necessário excluir as marisqueiras com dados faltantes. ${ }^{b}$ As características força e pressão física foram mensuradas em uma escala 0 a 5 (autorreferida), conforme o instrumento demanda física adaptado por Fernandes (2004)

* Diferença entre os grupos testada pelo qui-quadrado, significativa se $p<0,05$

A Tabela 2 demonstra todas as razões de prevalências deste estudo, desde as informações brutas, considerando todas as variáveis, bem como do modelo inicial até o final para o método escolhido. Verifica-se que as marisqueiras mais velhas tiveram $38 \%$ mais DME $\mathrm{P} / \mathrm{O}$ em relação às mais jovens RP: 1,38 , IC95\%: [1,14-1,64], conforme modelo final.

Ressalta-se que não houve interação entre as variáveis horas trabalhadas/dia e idade, conforme o modelo saturado apresentado na Tabela 2, respectivamente com valor de coeficiente igual a $-0,246$ e $p$-valor igual a 0,1807 . Desta forma não houve modificação do efeito na interdependência entre estas duas variáveis na ocorrência/prevalência de DME. Ainda neste contexto horas de trabalho/dia e idade quando concomitantes e ajustadas no modelo final se associam positivamente aos distúrbios musculoesqueléticos em região de pescoço ou ombro, entretanto não modificam o efeito no acréscimo ou diminuição.

As marisqueiras que trabalhavam mais que $11 \mathrm{~h}$ por dia tiveram uma $\mathrm{RP}=1,23$ com IC95\%: $[1,04 ; 1,45]$; $23 \%$ mais $\mathrm{DME}$ em relação às que trabalhavam menos que $11 \mathrm{~h}$. Foi evidenciada ainda a associação positiva, estatisticamente significante, de acordo com a presença de artrite $\mathrm{RP}=1,32 \mathrm{com}$ IC95\%: [1,12-1,56] e tempo de trabalho maior que 26 anos e DME P/O RP: 1,22, IC95\%: $[1,02-1,45]$, conforme demonstrado nas Tabelas 1 e 2. 
Tabela 2 - Razões de Prevalência (RP) e Intervalos de Confiança (IC) de 95\% dos modelos de Poisson realizados para DME em pescoço ou ombro ( $n=205$ ) em uma amostra de Marisqueiras de Saubara, BA,2013

\begin{tabular}{|c|c|c|c|c|c|}
\hline \multirow{2}{*}{ Variáveis } & \multicolumn{2}{|c|}{$\begin{array}{c}\text { Modelo Inicial }^{\text {a }} \\
\text { (a partir da análise bivariada) }\end{array}$} & \multicolumn{2}{|c|}{ Modelo final ${ }^{b}$} & \multirow{2}{*}{$\begin{array}{l}\text { Modelo Saturado }{ }^{c} \\
\text { Coeficiente } \\
\text { (valor de p) }\end{array}$} \\
\hline & $\begin{array}{l}\text { Coeficiente } \\
\text { (valor de p) }\end{array}$ & RP [IC95\%] & $\begin{array}{l}\text { Coeficiente (valor } \\
\text { de } p \text { ) }\end{array}$ & RP[IC95\%] & \\
\hline Horas de trabalho/dia & $\begin{array}{r}0,220 \\
(0,0082)\end{array}$ & - & $\begin{array}{r}0,207 \\
(0,0145)\end{array}$ & - & $\begin{array}{r}0,360 \\
(0,0252)\end{array}$ \\
\hline$<11 \mathrm{~h} / \mathrm{dia}$ & - & 1 & - & 1 & - \\
\hline$\geq 11 \mathrm{~h} / \mathrm{dia}$ & - & $1,25[1,06-1,47]$ & - & $1,23[1,04-1,45]$ & - \\
\hline Idade & $\begin{array}{r}0,397 \\
(0,0011) \\
\end{array}$ & - & $\begin{array}{r}0,319 \\
(0,0006) \\
\end{array}$ & - & $\begin{array}{r}0,369 \\
(0,0008) \\
\end{array}$ \\
\hline$<38$ anos & - & 1 & - & 1 & - \\
\hline$\geq 38$ anos & - & $1,49[1,17-1,89]$ & - & $1,38[1,15-1,65]$ & - \\
\hline Horas de trabalho/dia * Idade & - & - & - & - & $\begin{array}{r}-0,246 \\
(0,1807) \\
\end{array}$ \\
\hline Escolaridade & $\begin{array}{r}0,022 \\
(0,8536)\end{array}$ & - & - & - & - \\
\hline$\geq 2^{\circ}$ incompleto & - & 1 & - & - & - \\
\hline$<2^{\circ}$ incompleto & - & $1,02[0,81-1,29]$ & - & - & - \\
\hline Tempo de trabalho em anos & $\begin{array}{r}-0,153 \\
(0,1169) \\
\end{array}$ & - & - & - & - \\
\hline$\leq 26$ anos & - & 1 & - & - & 一 \\
\hline$>26$ anos & - & $0,86[0,71-1,04]$ & - & - & - \\
\hline Horas de trabalho doméstico/semana & - & 一 & - & - & - \\
\hline$\leq 7 \mathrm{~h}$ & - & - & - & - & - \\
\hline$>7 \mathrm{~h}$ & - & 一 & 一 & - & - \\
\hline IMC & - & - & - & - & - \\
\hline$\leq 24,9 \mathrm{Kg} / \mathrm{m}^{2}$ & - & - & - & - & - \\
\hline$\geq 25,0 \mathrm{Kg} / \mathrm{m}^{2}$ & - & 一 & 一 & - & - \\
\hline Atividade física & - & 一 & 一 & - & - \\
\hline $\operatorname{Sim}(\geq 3 x$ semana/30min) & - & 一 & - & - & 一 \\
\hline Não (< 3x semana/30min) & - & - & - & - & - \\
\hline Força & - & 一 & 一 & - & - \\
\hline$<2$ & - & - & - & - & - \\
\hline$\geq 2$ & - & - & - & - & - \\
\hline Pressão física & - & 一 & - & - & - \\
\hline$<2$ & - & - & & - & - \\
\hline$\geq 2$ & - & - & & - & 一 \\
\hline Demanda psicossocial & $\begin{array}{r}-0,103 \\
(0,2550) \\
\end{array}$ & - & - & - & - \\
\hline Baixa & - & 1 & - & - & - \\
\hline Alta & - & $0,90[0,76-1,07]$ & - & - & - \\
\hline Artrite & $\begin{array}{r}0,133 \\
(0,1054) \\
\end{array}$ & - & - & - & - \\
\hline Não & - & 1 & - & - & - \\
\hline Sim & - & $1,14[0,97-1,34]$ & - & - & - \\
\hline Diabetes & - & - & - & - & - \\
\hline Não & 一 & 一 & 一 & - & 一 \\
\hline Sim & - & - & - & 一 & - \\
\hline
\end{tabular}

Fonte: Dados da pesquisa.

Legenda: ${ }^{a}$ considerando variáveis que obtiveram um p-valor inferior a 0,20. AIC: 399,02; Deviance: 93,12; $p=1$

${ }^{b}$ considerando variáveis que obtiveram um p-valor inferior a 0,05. AlC: 395,68; Deviance: 93,68; $p=1$

${ }^{c}$ modelo de interação entre Horas de trabalho/dia e Idade. AIC: 393,84; deviance: 93,84; $p=1$

* $p<0,05$ no teste de Wald. 


\section{DISCUSSÃO}

Os resultados apresentados demonstraram que as marisqueiras investigadas convivem com doenças crônicas e suas repercussões, devido à elevada prevalência de DME em região de pescoço e ombro, na totalidade de ocorrência em $71,2 \%$ das trabalhadoras, revelando a gravidade dessa patologia para este contingente de trabalhadoras. Verificou-se que as pescadoras com idade superior a 38 anos apresentam $38 \%$ mais DME de pescoço ou ombro em comparação com as mais jovens.

Outro achado relevante, também em Saubara/BA, demonstra que as marisqueiras que trabalhavam mais que $11 \mathrm{~h}$ por dia tiveram $23 \%$ mais DME em relação às que trabalhavam menos que $11 \mathrm{~h}$ e denotam a degradação da saúde com a carga exaustiva de trabalho. A longa jornada de trabalho dessas pescadoras já foi relatada por Pena et al. (2011), em um estudo etnográfico.

Segundo Yeng et al. (2001), existe uma predisposição individual para o desenvolvimento de síndromes dolorosas crônicas. $O$ descondicionamento do aparelho cardiovascular e locomotor, a constituição física, as características sexuais, o perfil comportamental psíquico, o elevado grau de estresse e de insatisfação no ambiente de trabalho, familiar e social, o reforço da condição de incapacidade, a negação da condição de bem-estar e os ganhos e perdas pessoais são fatores que implicam a gênese e a perpetuação da sintomatologia. Segundo Pena, Freitas e Cardim (2011), para as pescadoras artesanais/marisqueiras, a exposição aos riscos inicia-se na infância, quando manejam instrumentos de trabalho, caminhando sob o sol, intempéries, sobre as pedras e mangues, sem qualquer proteção. Os riscos relativos aos fatores externos se acumulam ao longo da vida laboral e somam-se às condições que repercutem negativamente no seu crescimento

Ressalta-se que existem poucas evidências científicas demonstrando a relação de DME em pescadores; dentre estes, destaca-se o estudo realizado por Chang et al.(2012) que utilizou o Questionário nórdico, versão chinesa, nos pescadores de ostra em Taiwan, que apresentavam dor musculoesquelética nas costas, mãos, punhos, ombros e cotovelos. Em outro estudo, produzido por Berg-Beckhoff, Ostergaard e Jepsen (2016), que investigou os pescadores dinamarqueses, ficou ratificado que a pesca é fisicamente desgastante e gera distúrbios musculoesqueléticos.

É notório que diferente dos trabalhadores formais, a ausência no trabalho e a diminuição do ritmo (diminuição das horas trabalhadas) acarretariam a diminuição da produção, essencial para a sua subsistência. Ainda segundo Pena, Freitas e Cardim (2011), "[...] a condição do trabalho da marisqueira com a dor crônica se configura em um modo de vida", pois elas não deixam de trabalhar mesmo apresentando algum tipo de DME. Esta afirmação pode ser comprovada neste estudo, pois, mesmo com a elevada prevalência de DME entre essas trabalhadoras, a jornada de trabalho é extensa, exaustiva, em comparação com diversas outras categorias profissionais.

A constatação da magnitude do problema de saúde pública nas marisqueiras de Saubara/BA demonstra uma prevalência de DME de pescoço e ombro muito superior em comparação a outros estudos, tanto com pescadores artesanais, quanto com os profissionais formais. No estudo de Müller et al. (2016), evidenciou-se a presença de doenças crônicas não transmissíveis e a predominância de DME global (em todas as regiões do corpo), com uma prevalência de $94,7 \%$, seguida de hipertensão arterial $(27,3 \%)$, artrite $(10,5 \%)$ e diabetes $(6,7 \%)$.

Para Falcão et al. (2015) ficou demonstrada a prevalência elevada de DME em pescoço ou ombro e em membros superiores distais, nas marisqueiras de Sauba$\mathrm{ra}$, e eles ainda ratificaram que a quase a totalidade das marisqueiras referiram dor ou desconforto em alguma parte do corpo, no último ano. Entretanto, não foi realizada relação com as horas dedicadas ao trabalho, como neste estudo.

Ainda esta mesma população revelou que os DME em pescoço ou ombro se associaram com as demandas físicas referentes aos fatores específicos do trabalho, principalmente com a etapa da coleta do marisco (apoiando-se sobre o cotovelo, apoiando-se sobre o punho, tronco rodado, movimentos precisos e muito finos com as mãos, empurrar e puxar a carga). Ratificou-se ainda que aquelas que variavam mais a postura, manuseio de carga, força muscular e pressão física, apresentaram menos DME nas duas unidades funcionais relatadas (FALCÃO et al., 2017).

A possível associação de DME pescoço/ombro com horas dedicadas ao trabalho pode, ao longo do tempo, gerar dor e redução da mobilidade articular e, em consequência, afetar negativamente as habilidades funcionais, as atividades de trabalho e a qualidade de vida das pessoas (MOUSAVI et al., 2008). Além disso, as afecções nessa articulação representam um acentuado problema socioeconômico, no caso das pescadoras artesanais, pois a manutenção da saúde é necessária para a execução do trabalho que gera a subsistência familiar.

Para Moraes et al. (2009), a incapacidade de membros superiores de trabalhadores que expõem esse complexo articular em grande repetitividade de movimentos e posicionamento dos membros superiores em abdução do ombro está associada, principalmente, à fadiga muscular, a fatores de organização do trabalho, à anulação do mecanismo de regulação do trabalho, com consequente sobrecarga (diminuição ou falta de intervalo), e a fatores psicossociais que acarretam tensão e estresse.

A confirmação de distúrbio musculoesquelético nos membros superiores representa um importante problema socioeconômico e, no caso de pescadoras artesanais, de maneira acentuada, pois a manutenção da saúde é necessária para a execução do trabalho que gera a subsistência familiar. 


\section{CONCLUSÃO}

O presente artigo encontrou uma elevada prevalência de DME em pescoço/ombro nas marisqueiras mais velhas, que trabalhavam mais de 11 horas por dia, bem como associação positiva e estatisticamente relevante na presença de artrite. Neste contexto, é necessária a meIhoraria da atenção à saúde dessa categoria profissional, gerando decisões e medidas específicas voltadas para suas condições médicas crônicas.

\section{AGRADECIMENTOS}

Ao grupo de pesquisa Saúde, Meio-Ambiente e Sustentabilidade da Pesca Artesanal (PROSAS/UFBA) e todas as pescadoras artesanais / marisqueiras do município de Saubara, Bahia. Conforme a portaria no 206, de 4 de setembro de 2018. O presente trabalho foi realizado com apoio da Coordenação de Aperfeiçoamento de Pessoal de Nível Superior - Brasil (CAPES) - Código de Financiamento 001, bem como à FAPESB pelo apoio e incentivo no desenvolvimento desta pesquisa.

\section{REFERÊNCIAS}

ALMEIDA, P.C. A.; BARBOSA-BRANCO, A. Acidentes de trabalho no Brasil: prevalência, duração e despesa previdenciária dos auxílios-doença. Rev. Bras. Saúde Ocup., São Paulo, v.36, n.124, p.195-207, 2011. DOI: http:// dx.doi.org/10.1590/S0303-76572011000200003.

ANDERSEN, J.H.; HAAHR, J.P.; FROST, P. Risk factors for more severe regional musculoskeletal symptoms: a two-year prospective study of a general working population. Arthritis rheum., Atlanta, v. 56, n.4, p.1355-1364, 2007.

ARAÚJO, T.M.; KARASEK, R. Validity and reliability of the job content questionnaire in formal and informal jobs in Brazil. Scand. j. work environ. health, Helsinki, v.6, p.52-59, 2008.

ARMSTRONG, T.J. et al. A conceptual model for work-related neck and upper-limb muscoloskeletal disorders. Scand. j. work environ. health, Helsinki, v.19, n.2, p.73-84,1993.

BERG-BECKHOFF, G.; OSTERGAARD, H.; JEPSEN, J.R. Prevalence and predictors of musculoskeletal pain among Danish fishermen - Results froma cross-sectional surve. J. Occup. Med. Toxicol., London, v.11, 2016.

BONGERS, P.M. et al. Epidemiology of work related neck and upper limb problems: psychosocial and personal risk factors (part I) and effective interventions from a bio behavioural perspective (part II). J. occup. rehabil., New York, v.16, n.3, 272-295, 2006.

BRASIL. Ministério da Pesca e Aquicultura. Planos e políticas. Brasília: MS, 2012.

. Ministério da Saúde. Diagnóstico, tratamento, reabilitação, prevenção e fisiopatologia das LER/DORT: procedimentos. Brasília: MS, 2001.

Ministério da Saúde. Protocolos de Atenção Integral à Saúde do Trabalhador de Complexidade Diferenciada. Lesões por esforços repetitivos (LER). Distúrbios osteomusculares relacionados ao trabalho (Dort). Dor relacionada ao trabalho. Brasília:MS, 2006.

CHANG, J. H. et al. Prevalence of musculoskeletal disorders and ergonomic assessments of cleaners. Am. j. ind. med., New York, v. 55, p. 593-604, 2012.
DEVEREUX, J. J.; VLACHONIKOLIS, G.; BUCKLE, P. W. Epidemiological study to investigate potential interaction between physical and psychosocial factors at work that may increase the risk of symptoms of musculoskeletal disorder of the neck and upper limb. Occup. environ. med. London, v. 59, n. 4, p. 269-277, 2002.

FALCÃO, I. R. et al.Fatores associados com os distúrbios musculoesqueléticos em pescadoras artesanais/marisqueiras em Saubara, Bahia, Brasil. Ciênc. Saúde Colet., Rio de Janeiro, out. 2017. Disponível em: <http:// www.cienciaesaudecoletiva.com.br/artigos/fatores-associados-com-os-disturbios-musculoesqueleticos-em-pescadoras-artesanaismarisqueiras-em-saubara-bahia-brasil/16443>. Acesso em: 15 jan. 2017.

FALCÃO, I. R. et al. Prevalência dos distúrbios musculoesqueléticos nos membros superiores e pescoço em pescadoras artesanais/marisqueiras em Saubara, Bahia, Brasil. Ciênc. Saúde Colet., Rio de Janeiro, v. 20, n.8, p.2469-2480, 2015. Disponível em: http://dx.doi.org/10.1590/141381232015208.17272014. Acesso em: 4 mar. 2017.

FOOD AND AGRICULTURAL ORGANIZATION. The state of world fisheries and aquaculture 2016: Contributing to food security and nutrition for all. Rome, 2016. 200 p. Disponível em: <www.fao.org/3/a-i5555e.pdf>. Acesso em: 4 mar. 2017.

FERNANDES, R. C. P. Distúrbios musculoesqueléticos e trabalho industrial. 2004. Tese (Doutorado em Saúde Coletiva) - Universidade Federal da Bahia, Salvador.

FERNANDES, R.C.P.; CARVALHO, F. M.; ASSUNÇÃO, A. A. Prevalence of musculoskeletal disorders among plastics industry workers. Cad. saúde pública, Rio de Janeiro, v. 27, n.1, p. 78-86, 2011.

FERNANDES, R.C.P. et al. The concurrence of musculoskeletal pain and associated work-related factors: a cross sectional study. BMC public health, London, v.16, p. 628, 2016. DOI:10.1186/s12889-016-3306-4.

KARASEK, R. Job content instrument: questionnaire and user's guide. Massachusetts: University of Massachusetts; Amherst, 1985.

KARASEK, R. et al. The Job Content Questionnaire (JCQ): an instrument for internationally comparative assessments of psychosocial job characteristics. J. occup. health psychol., Washington, v.3, n. 4, p.322-355, 1998.

KUORINKA, I. et al. Standardised Nordic questionnaires for the analysis of musculoskeletal symptoms. Appl .ergon., Oxford, v.18, n.3, p.233237, 1987.

KUORINKA, I.; FORCIER, L. Work related musculoskeletal disorders (WMSDs): a reference book for prevention. London: Taylor \& Francis, 1995.

LIN, T.Y. et al. Distúrbios ósteo-musculares relacionados ao trabalho. Rev. Med., [s.I], v. 80, p. 422-442, 2001.

MACKINNON, J. G.; WHITE, H. Some heteroskedasticity-consistent covariance matrix estimators with improved finite sample properties. J Econom., Amsterdam, v. 29, p. 305-325, 1985.

MANDEL, M. Simulation based confidence intervals for functions with complicated derivatives. The American Statistician, v.67, n.2, p.76-81, 2013.

MORAES, G.F.S. et al. Correlação entre posicionamento escapular, análise postural funcional e grau de incapacidade dos membros superiores (DASH Brasil) em médicos ultrassonografistas. Radiol. Bras., São Paulo, v.42, n.1, 2009. Disponível em: <http://www.scielo.br/scielo. php?script=sci_arttext\&pid=S0100-39842009000100008\&lng=en\&nr m=iso>. Acesso em: 20 fev. 2015.

MOUSAVI, S.J. et al. Cultural adaptation and validation of the Persian version of the Disabilities of the Arm, Shoulder and Hand (DASH) 
outcome measure. Clin. rehabil., London, v.22, n. 8, p.749-757, 2008.

MÜLLER, J. S. et al. Health-related quality of life among artisanal fisherwomen/shellfish gatherers: Lower than the general population. Int. J. environ. res. public health., Basel, v. 3, n.5, p.1-12, 2016.

NATIONAL RESEARCH COUNCIL. INSTITUTE OF MEDICINE. Commission on behavioral and social sciences and education. Musculoskeletal disorders and the workplace: Low back and upper extremities. Panel on musculoskeletal disorders and the workplace. Washington: National Academy Press, 2001.

PENA, P.G.L.; FREITAS, M.C.S.; CARDIM, A. Trabalho artesanal, cadências infernais e lesões por esforços repetitivos: estudo de caso em uma comunidade de mariscadeiras na Ilha de Maré, Bahia. Ciênc. Saúde Colet., Rio de Janeiro, v.16, n. 8, p. 3383-3392, 2011.

PUNNETT, L.; WEGMAN, D. H. Work-related musculoskeletal disorders: the epidemiologic evidence and the debate. J Electromyogr. Kinesiol., New York, n.14, 2004.

R CORE TEAM. R: A language and environment for statistical computing. Vienna: R Foundation for Statistical Computing, 2018. Disponível em: <https://www.R-project.org/>. Acesso em: 20 fev. 2015.
RIOS, A.O.; REGO, R.C.F.; PENA, P.G.L. Doenças em trabalhadores da pesca. Rev. baiana de saúde pública, Salvador, v.35, n.1, p.175-188, 2011.

SAKAMOTO, Y.; ISHIGURO, M.; KITAGAWA, G. Akaike Information Criterion Statistics. Boston: D. Reidel Publishing Company, 1986.

ZOU, G. A modified poisson regression approach to prospective studies with binary data. Am. J. Epidemiol., Baltimore, v.159, n.7, p. 702-706, 2004.

WANG, P. C. et al. Work-organisational and personal factors associated with upper body musculoskeletal disorders among sewing machine operators. Occup. environ med., London, v. 64, n.12, p. 806-813, 2007.

WORLD HEALTH ORGANIZATION. Identification and control of work-related diseases. Report of a WHO Expert Committee. Genebra: WHO, 1985.

WORLD HEALTH ORGANIZATION. The international classification of adult underweight, overweight and obesity according to BMI. Genebra:WHO, 2004.

YENG, L. T. et al. Distúrbios ósteo-musculares relacionados ao trabalho. Rev Med., [s.I], v. 80, p. 422-442, 2001.

Submetido em: 01/02/2019

Aceito em : 30/04/2019 